\title{
A Comparison between Two Kidney Transplant Ureteroneocystostomy Techniques: Full-Thickness Single Layer Anastomosis and Lich-Gregoir
}

\section{Abstract}

Urinary complications such as urine leak, stricture, ureteric obstruction and vesicoureteral reflux are the most common technical adverse events following A variety of urinary tract reconstructive methods have been proposed and compared to find an ideal method with minimum urinary complications. Today, ureteroneocystostomy is the most common urinary tract reconstructive method utilized in kidney transplantation. The main aim of the present study was to compare urologic complications following the use of FTSL and LG techniques in kidney transplant ureteroneocystostomy in a clinical trial study.

Keywords: Urinary complications; Kidney transplantation; Ureteroneocystostomy

Received: July 13, 2016; Accepted: August 04, 2016; Published: August 10, 2016

\section{Introduction}

Urinary complications such as urine leak, stricture, ureteric obstruction and vesicoureteral reflux are the most common technical adverse events following Kidney transplantation which may be associated with significant morbidity, excess costs and need for reoperation [1,2]. Overall, the incidence of urinary complications has been reported to vary from $4 \%$ to $23 \%$ in different studies [2-5]. The majority of these complications are related to vesicoureteral implantation site $[6,7]$. A variety of urinary tract reconstructive methods have been proposed and compared to find an ideal method with minimum urinary complications and better surgical characteristics [2-4,7-12]. Today, ureteroneocystostomy is the most common urinary tract reconstructive method utilized in kidney transplantation $[3,9,10,13]$ and divided to extravesical and intravesical methods and on the other hand techniques that employ the antireflux mechanisms and those who don't [2].

Lich-Gregoir (LG) is a commonly used extravesical ureteroneocystostomy technique in which the ureter is sutured to the mucosal layer of the bladder and detrusor musculature closes over the implanted ureter to function as an antireflux mechanism [2,4,7-9]. In recent years at our center, along with the LG technique a lesser known extravesicle ureteroneocystostomy technique has been utilized in which the ureter is continuously sutured directly to the full thickness of the bladder without any additional closure of the musculature to serve as an antireflux mechanism. To the best of our knowledge, a limited number

\section{Behnam Sanei ${ }^{1}$, Mozaffar Hashemi ${ }^{1}$, Abbas Tabataei ${ }^{1}$, Razie Jafari ${ }^{2}$ and Hamid Reza Jafari ${ }^{3}$}

Department of Transplant Surgery, Isfahan University of Medical Sciences, Isfahan, Iran

2 Department of Surgery, Isfahan University of Medical Sciences, Isfahan, Iran

3 School of Medicine, Isfahan University of Medical Sciences, Isfahan, Iran

Corresponding author: Razie Jafari

rjafari900@gmail.com

Department of Surgery, Isfahan University of Medical Sciences, Isfahan, Iran.

Tel: +989132840164

Citation: Sanei B, Hashemi M, Tabataei A, et al. A Comparison between Two Kidney Transplant Ureteroneocystostomy Techniques: Full-Thickness Single Layer Anastomosis and Lich-Gregoir. J Univer Surg. 2016, 4:3.

of studies are available on this previously named full thickness (FT) method. According to the previous reports (retrospective analysis from University of Florida) this technique is associated with lower incidence of urine leak and a similar incidence of other urological complications(including reflux) compared to the LG technique during the first year post operatively $[2,12]$. On the other hand, the FTSL is a technically simpler method compared to the LG technique [12].

The aim of the present study was to compare urologic complications following the use of FTSL and LG techniques in kidney transplant ureteroneocystostomy in a clinical trial study.

\section{Methods and Patients}

This prospective, randomized, clinical trial was conducted between November 2012 and November 2013 in Al-Zahra Educational Hospital (Isfahan, Iran) affiliated to Isfahan University 
of Medical Sciences. The study was registered in Iranian Registry of Clinical Trials (Registration code: IRCT2013123116013N1) and the study protocol was reviewed and approved by the ethical committee of the same university (project number: 3931960). Written informed consents were obtained from the participants.

The patients who were hospitalized to undergo kidney transplant operation at Al-Zahra Hospital were screened for inclusion. Adult patients (aged $>18$ years), who were scheduled to undergo isolated kidney transplantation, and did not have any contraindication for surgery were included in this study. Exclusion criteria included occurrence of renal failure, primary nonfunction (the allograft never starts to function and results in the permanent post transplantation requirement for dialysis) or graft loss during the follow-up period. Sixty patients were randomly assigned either to the LG $(\mathrm{N}=30)$ or to the FTSLA $(\mathrm{N}=30)$ groups based on computer generated random sequence. The patients' preparation, anesthesia and surgical procedure were the same for the two groups except for the ureteroneocystostomy technique employed. All transplants were obtained from cadaveric donors.

The preparation of bladder and ureter was the same in both groups. In male recipients, the ureter was brought under the spermatic cord to a convenient position on the anterolateral bladder wall (in females, the round ligament of the uterus was divided). In the FTSL group, an incision (cystostomy) was made through all layers of the bladder wall (mucosa, musculature, and serosa) over $6 \mathrm{~mm}$ to $8 \mathrm{~mm}$. An anti-mesenteric incision was then made on the tip of the donor ureter to spatulate it. The ureteral end was continuously sutured to the full thickness (all layers) of the bladder using 5/0 polydioxanone (PDS) sutures (Dogsan Ltd., Istanbul, Turkey). In the LG group a $2 \mathrm{~cm}$ to $3 \mathrm{~cm}$ incision was made in the bladder wall muscle at the dome until the mucosa of the bladder wall was exposed. Then, the bladder mucosa was opened $1 \mathrm{~cm}$ at the distal angle of the incision and sutured to the ureteral end with continuous 4/0 vicryl sutures (Dogsan Ltd., Istanbul, Turkey). The bladder (detrusor) muscle and serosa were then closed over the anastomosis using separate $2 / 0$ vicryl sutures (Dogsan Ltd., Istanbul, Turkey) to make a submucosal tunnel as an antireflux mechanism. A magnifying loupe (2.5X) was used during the ureteroneocystostomy procedure. Ureteral stents (a 6 French ( $\mathrm{CHO6}$ ) feeding tube) were used for all transplants and were removed by a cystoscopy 6 weeks after surgery. In both groups a Jackson-Pratt (JP) drain was placed through the lower quadrant of the abdomen adjacent to the surgical site. The drain was removed once the drainage became less than $25 \mathrm{ml} /$ day and maximally 2 weeks post operatively. All of the transplants in both groups were performed by the same surgical team. The patients were transferred to the recovery unit and then to the transplant ward. Foley catheters were removed 3-4 days postoperatively. The creatinine $(\mathrm{Cr})$ level of drain content was checked daily and compared to the serum creatinine for 2 weeks. Urine leak was defined as drainage $\mathrm{Cr} /$ serum $\mathrm{Cr}$ higher than 6 [14]. At $6^{\text {th }}$ month postoperatively the patients underwent ultrasonography of the kidney, bladder and ureter for diagnosis of ureteral obstruction and voiding cystourethrograms (VCUG) was performed to assess the reflux and stenosis. The patients' medication including the immunosuppressive regimen, cytomegalovirus prophylaxis and antibiotic prophylaxis was similar for both groups which were administered under the supervision of a nephrologist. All data were recorded by the same physician not otherwise involved in the study and blinded to the surgical technique employed.

All statistical analyses were performed using the Statistical Package of Social Sciences (SPSS) version 19.0 (SPSS, Chicago, IL, USA). Categorical data was analyzed using the Fisher's exact test. Parametric data was analyzed using the unpaired t-test. Descriptive statistics were expressed as mean \pm standard deviation or number (percentage in parenthesis). $P$ value less than 0.05 was considered statistically significant.

\section{Results}

Sixty-four patients were assigned to two groups, each with 32 patients. Five patients in the LG group were excluded (one patient underwent nephrectomy because of renal artery thrombosis, one patient was expired and three patients suffered from primary non-function of allograft) and three patients in the FTSL group (one underwent nephrectomy because of renal artery thrombosis and two patients suffered from primary non-function of allograft). Fifty-six patients with the mean age of $41.16 \pm 14.32$ years (ranged 18 to 75 years) were included in final analysis of this study (27 in LG and 29 in FTSL group). No statistically significant differences were noted between the FTSL and the LG group in terms of the mean male/female ratio, age, weight and height of the patients $(p>0.05)$ (Table 1$)$.

The incidence of the diagnosed urine leak, stricture, ureteric obstruction and VUR has been presented in Table 2 . The Overall incidence of these 4 urinary complications was 33\% in the LG group and $10.3 \%$ in the FTSL group. The Fisher's exact test showed no significant difference between the two groups regarding the incidence of each of the mentioned complications $(p>0.05)$ (Table 2).

\section{Discussion}

In this study we compared clinical outcomes of the FTSL (lesserknown technique) and the LG technique (the more popular one) in kidney transplant ureteroneocystostomy. This investigation showed that urine leakage and stenosis occurred slightly less frequently in the patients who underwent the FTSL in comparison to those in the LG group. However, there was no statistical significance.

Since 1954 when the first successful kidney transplant was performed by Harrison et al. [15], various ureteral reimplantation techniques have been proposed in the literature. The first techniques were interavesical that used two cystotomies, one as the graft ureter implantation sit and the other to access the interior of the bladder. The first extravesical method was described

Table 1: Demographic data.

\begin{tabular}{|c|c|c|c|}
\hline & Lich-Gregoir & Full-thickness single layer & P value \\
\hline Number & 27 & 29 & - \\
\hline Age (year) & $42.48 \pm 14.78 *$ & $39.93 \pm 14.02$ & 0.511 \\
\hline Weight (kg) & $67.70 \pm 8.41$ & $66.69 \pm 9.83$ & 0.681 \\
\hline Height (cm) & $170.41 \pm 8.98$ & $169.38 \pm 8.47$ & 0.661 \\
\hline \multicolumn{2}{|c|}{ *Data are expressed as mean \pm standard deviation } \\
\hline
\end{tabular}


Table 2: Rate of complications in two groups.

\begin{tabular}{|c|c|c|c|}
\hline & Lich-Gregoir & Full-thickness single layer & P Value \\
\hline Leak & $3(11.1 \%)^{*}$ & 0 & 0.106 \\
\hline Obstruction & 0 & 0 & - \\
\hline Stenosis & $4(14.8 \%)$ & $1(3.4 \%)$ & 0.185 \\
\hline Reflux & $2(7.4 \%)$ & $2(6.9 \%)$ & 0.667 \\
\hline & *Data are expressed as number (\%) \\
\hline
\end{tabular}

by Witzel [16] and, then by Gregoir [17] and soon after by Lich [18]. This technique was designed in order to avoid a separate cystostomy and is related to lower complication rate [19]. Several variations of this method have been introduced. The LG technique is characterized by the formation of a submucosal anti-reflux tunnel and a urothelial anastomosis. Anti-refluxive techniques were developed to prevent VUR, but non-anti-reflexive methods were proposed based on the idea that VUR occurs despite the anti-reflux tunneling procedure and this asymptomatic reflux is not very important when the bladder and ureter are normal.

In 2010, Kayler et al. [2] in a review of literature described a simple ureteroneocystostomy method with a full-thickness ureter to bladder anastomosis employed by some surgeons at the University of Florida and named it as FT method. They reviewed the records of 126 adult renal transplant recipients who underwent FT ureteroneocystostomy method between April 2007 and September 2008 with a one-year follow-up. One case with gross hematuria necessitating bladder irrigation, 3 cases of ureteral strictures, and neither urinary leakages nor symptomatic VUR necessitating operative intervention were reported [2]. Kely et al. [2] also compared the outcomes of FT and LG techniques in 634 renal transplant recipients ( 307 LG and 327 FT) between December 2006 and December 2010 in the same university. They reported a similar incidence of urinary complications for the two groups [12]. Contrary to the aforementioned studies we performed our study prospectively (as a clinical trial) and the same surgical team performed all of the transplants. We performed all evaluation tests on all of the patients at the determined time regardless of signs and symptoms.

In this study, the incidence of urine leak was $11 \%$ with the LG technique that is comparable with the range reported ( $0 \%$ to $8.2 \%$ ) by previous studies $[7,10,11,20]$. Our result with the FTSL technique is consistent with that of Kayler et al.'s [2] study which reported an incidence of $0.6 \%$ for urine leak with the FTSL technique [12]. Urine leak is a relatively rare complication presumably associated with technical issues [12]. Leakage occurs from anastomosis site; ureter or the renal pelvis due to ureteral necrosis resulting from insufficient blood supply caused by extensive coagulation of the ureter, periureteral fat stripping during procurement or increased urinary pressure during obstructions. In the LG technique when the bladder mucosa is extremely thin the anasthomosis could be complicated as extravasation of urine through suture orifices or tearing of mucosa away from the suture lines $[10,12]$. But in the
FTSL technique, involvement of the full thickness of the bladder wall in sutures results in a strong sealing and lowers the potential for urine leakage.

The results of our study showed that ureteral stenosis occurred in $4(14.8 \%)$ cases in the LG group and one $(3.4 \%)$ in the FTSL group. Previous studies reported an incidence rate of $2.3 \%$ to $5.3 \%$ for stenosis with the LG technique $[7,10]$. It occurs almost always at $6^{\text {th }}$ month postoperatively and may occur in any location [3]. The incidence of ureteral obstruction has been reported to range from $1 \%$ to $5.5 \%[10,11,20]$.The ureteral obstruction might be extrinsic or intrinsic [7]. Intrinsic (intraluminal) obstruction of the ureter can be caused by conditions like stricture, hematoma or stenosis. Extrinsic (extraluminal) obstruction of the ureter can be caused by adhesions, collections, or masses [21]. It seems that LG method might be more prone to develop obstruction because of kinking within the submucosal tunnel. But in this study the patients developed obstruction neither in the LG nor in the FTSL group.

Two (7\%) cases with VUR were diagnosed in each group. Kayler et al. [2] did not assess the frequency of VUR in their retrospective study as only two patients had undergone testing for VUR [12]. The submucosal tunnel has been recommended in some methods (including LG) as an anti-reflux mechanism. However, the incidence of VUR ranges from $2 \%$ to $86 \%$ when routinely assessed. Therefore, the asymptomatic VUR is a common complication which occurs despite the submucosal tunneling and is not as important as once thought [7]. On the other hand, it has been shown that the probability of VUR increases with posttransplant time regardless of the surgical technique employed. In this study we used a so called refluxive technique and the incidence of VUR was the same in the two groups while the FTSL is a simpler surgical technique. In addition, it is associated with lower incidence of other complications including leakage ( $0 \%$ vs. $11 \%$ ) and stenosis ( $3.4 \%$ vs. $14.8 \%$ ), which, although not statistically significant, is clinically important to us. A significant difference is yet to be demonstrated by further studies with larger sample sizes. Another limitation in our study was that we did not assess other complications such as urinary tract infection and complicated hematuria.

\section{Conclusion}

This study shows a statistically non-significant but clinically important lower urological complication rate with FTSLA compared to the LG technique. Therefore, this technique could be considered as an option in kidney transplant ureteroneocystostomy. Further studies with larger sample size are recommended.

\section{Funding and Support}

This research was supported by the Research Department of Isfahan University of Medical Sciences. 


\section{References}

1 Englesbe MJ, Dubay DA, Gillespie BW, Moyer AS, Pelletier SJ, et al. (2007) Risk factors for urinary complications after renal transplantation. Am J Transplant 7: 1536-1541.

2 Kayler L, Kang D, Molmenti E, Howard R (2010) Kidney transplant ureteroneocystostomy techniques and complications: review of the literature. Transplant Proc 42: 1413-1420.

3 Fallah MR, Taghizadeh AA, Sharafi AH (2011) Technical modifications of ureteroneocystostomy in renal transplantation: an 18-year experience. Saudi J Kidney Dis Transpl 22: 1121-1127.

4 Lee RS, Bakthavatsalam R, Marsh CL, Kuhr CS (2007) Ureteral complications in renal transplantation: a comparison of the Lich-Gregoir versus the Taguchi technique. Transplant Proc 39: 1461-1464.

5 Veale JL, Yew J, Gjertson DW, Smith CV, Singer JS, et al. (2007) Long-term comparative outcomes between 2 common ureteroneocystostomy techniques for renal transplantation. J Urol 177: 632-636.

6 Moreno-Alarcón C, López-Cubillana P, López-González PÁ, PrietoGonzález A, Ruiz-Morcillo JC, et al. (2014) Lich-Gregoir technique and routine use of double $J$ catheter as the best combination to avoid urinary complications in kidney transplantation. Transplant Proc 46: 167-169.

7 Baston C, Harza M, Preda A, Gener I, Manea I, et al. (2014) Comparative urologic complications of ureteroneocystostomy in kidney transplantation: transvesical Leadbetter-Politano versus extravesical Lich-Gregoir technique. Transplant Proc 46: 176-179.

8 Moreira P, Parada B, Figueiredo A, Maia N, Nunes P, et al. (2007) Comparative study between two techniques of ureteroneocystostomy: Taguchi and Lich-Gregoir. Transplant Proc 39: $2480-2482$.

9 Pan XM, Xiang HL, Ding CG, Luo ZZ, Tian PX, et al. (2014) Comparative study of one-stitch versus Lich-Gregoir ureterovesical implantation for kidney transplants. World J Urol 32: 1355-1361.

10 Secin FP, Rovegno AR, Marrugat RE, Virasoro R, Lautersztein GA, et al. (2002) Comparing Taguchi and Lich-Gregoir ureterovesical reimplantation techniques for kidney transplants. J Urol 168: 926-930.
11 Zargar MA, Shahrokh H, Mohammadi Fallah MR, Zargar H (2005) Comparing Taguchi and anterior Lich-Gregoir ureterovesical reimplantation techniques for kidney transplantation. Transplant Proc 37: 3077-3078.

12 Kayler L, Zendejas I, Molmenti E, Chordia P, Schain D, et al. (2012) Kidney transplant ureteroneocystostomy: comparison of fullthickness vs. Lich-Gregoir techniques. Clin Transplant 26: E372-E380.

13 Saidi RF, Elias N, Hertl M, Kawai T, Cosimi AB, et al. (2013) Urinary reconstruction after kidney transplantation: pyeloureterostomy or ureteroneocystostomy. J Surg Res 181: 156-159.

14 Flores-Gama F, Bochicchio-Riccardelli T, Mondragón-Ramírez G (2010) Determination of creatinine in drained liquid. Urinary leak or lymphocele? Cir Cir 78: 327-332.

15 Harrison JH, Merrill JP, Murray JE (1956) Renal homotransplantation in identical twins. Surg Forum 6: 432-436.

16 Witzel O (1986) Extraperitoneal ureterocystostomie with oblique channel formation. Zentralbl Gynakol 20: 296-303.

17 Gregoir W (1962) Congenital vesico-ureteral reflux. Acta Urol Belg 30: $286-300$.

18 Lich R, Hoverton LW, Davis LA (1961) Recurrent urosepsis in children. J Urol 86: 554.

19 Slagt IK, Klop KW, ljzermans JN, Terkivatan T (2012) Intravesical versus extravesical ureteroneocystostomy in kidney transplantation: a systematic review and meta-analysis. Transplantation 94: 11791184.

20 Hakim NS, Benedetti E, Pirenne J, Gillingham KJ, Payne WD, et al. (1994) Complications of ureterovesical anastomosis in kidney transplant patients: the Minnesota experience. Clin Transplant 8: 504-507.

21 Nie Z, Zhang K, Huo W, Li Q, Zhu F (2010) Comparison of urological complications with primary ureteroureterostomy versus conventional ureteroneocystostomy. Clin Transplant 24: 615-619. 